DOI: https://doi.org/10.46630/phm.12.2020.15

Milana L. Dodig ${ }^{1}$

Article de recherche

Université de Kragujevac

УДК 811.133.1’366.58

Faculté des Lettres et des Arts

Reçu : le 10/1/2020

Département de langue et de littérature françaises

\title{
LE PHÉNOMÈNE D'ITÉRATION DANS L'ÉPOQUE PASSÉE EN FRANÇAIS ET LA QUESTION DE SA TRADUCTION
}

Ce travail traite du sujet de l'itération en tant qu'objet linguistique. Dans un premier temps, nous accorderons l'attention à la question du statut de l'itération en général, et notamment de la source de l'itération. Deuxièmement, il s'agira de montrer et d'analyser comment le français nous permet d'exprimer la répétition d'un procès dans le passé. Finalement, nous porterons notre intérêt sur la question de la traduction des exemples, issus de notre corpus dénotant l'itération passée, en français et en serbe. Cette approche nous permettra de dévoiler les nouveaux faits linguistiques concernant l'itération et l'itération dans l'époque passée, alors que l'analyse du corpus équivalent dans les deux langues révélera d'autres possibilités de l'expression de l'itération dont il n'en est pas fait de mention jusqu'à présent.

Mots-clés : itération, passé, aspect, temps, traduction.

\section{Introduction}

Dans la linguistique française l'itération est considérée comme une catégorie aspectuelle, traditionnellement nommée aspect itératif marquant la répétition d'un procès qui s'oppose à l'aspect semelfactif ${ }^{2}$ dénotant un procès ponctuel ne se produisant qu'une fois (RIEGEL, PELLAT et al. 1994 : 295). Aussi, faut-il souligner que le phénomène d'itération est étroitement lié à la nature lexicale et morphologique d'un verbe et à sa forme (i.e. de savoir à quel temps le verbe est conjugué). Pour une explication plus précise, citons M. Wilmet.

Dans le cadre de sa classification des aspects, cet auteur distingue les aspects lexical (fréquentatif) et affixal (duplicatif et multiplicatif) qui désignent la répétition d'une action. L'aspect fréquentatif indique « la fréquence du

\footnotetext{
${ }^{1}$ dodigmilana@filum.kg.ac.rs

${ }^{2}$ et à l'aspect singulatif selon la classification de Tournadre (2014) qui tient à distinguer ces deux aspects traditionnellement compris comme des équivalents.
} 
procès $\alpha-\omega$ ou le nombre de fois que se réalise le procès du terminus a quo $\alpha$ au terminus ad quem $\omega$ » et se réfère aux adverbes et aux circonstants de type : souvent, toujours, ne pas/jamais, une (deux, trois, quatre...) fois qui entrent en combinaison aspectuelle du verbe pour exprimer l'itération.

Les aspects duplicatif et multiplicatif concernent les affixes ; le premier est lié aux préfixes :

- re- « a une fonction duplicative intermittente : refaire ou redire bissent le procès $\alpha-\omega »$;

alors que le deuxième est lié aux infixes :

- aill- (p. ex. crier/criailler), -ass- (p. ex. rêver/rêvasser), -el- (p. ex. craquer/ craqueler), -ill- (fendiller), -in- (pleuviner), -nich- (pleurnicher), -och(bavocher), -on- (chantonner), -ot-(clignoter), -ouill- (mâchouiller) répètent le procès $\alpha-\omega$. (WILMET 2003 : 343-344)

Ensuite, il est des verbes en français, comme par ex. rabâcher, dont la nature lexicale signale directement l'itération, i.e. dont le contenu sémantique pose l'interprétation itérative comme centrale.

Quant à la relation l'itération-la forme d'un verbe, il est à noter que toutes les grammaires traditionnelles font mention de l'imparfait itératif (WILMET 2003 : 415) ou d'habitude (RIEGEL, PELLAT et al. 1994 : 308) :

(1) Chaque matin Paul allait chez le boulanger.

Aussi, l'itération représente la valeur que peut prendre le passé composé également :

(2) Longtemps, je me suis couché de bonne heure. (M. Proust, À la recherche du temps perdu)

Constatons que l'on peut obtenir l'interprétation itérative moyennant les adverbes et les circonstants dits fréquentatifs, la dérivation lexicale, le sémantisme de certains verbes et à l'aide de certains temps verbaux.

Par la suite, nous allons expliquer notre position à l'égard de ce traitement du phénomène d'itération, comme une valeur de tous les éléments mentionnés supra et surtout des temps verbaux, qui s'en distingue significativement.

D'abord, selon nous, l'itération, en tant que catégorie linguistique, représente un cas particulier de la répétition, « la répétition dans le temps d'un même procès » (GOSSELIN, MATHET et al. 2013 : 16). Nous nous appuyant sur les travaux de Bres $(2006,2007)$, nous posons que l'itération ne représente pas l'ingrédient d'un temps verbal ni des autres marqueurs traditionnellement tenus pour itératifs dont on a parlé supra. Ce sont les temps verbaux (« L'IMP, le $\mathrm{PS}$ et le $\left.\mathrm{PqP}^{3} »\right)$ qui sont « des ingrédients, parmi d'autres, de la production

\footnotetext{
${ }^{3}$ Bres utilise ces abréviations pour marquer, respectivement, l'imparfait, le passé simple et le
} 
des fréquences semelfactive et itérative » (BRES 2007 : 42). Ensuite, la plupart des travaux portant sur le préfixe re- constatent la grande complexité de ses valeurs sémantiques (LÉGER 1956 ; MOK 1964, 1980 ; JALENQUES 2001, cité par APOTHÉLOZ 2005 : 48) et même les différentes manifestations de son itérativité (ibid.). Bref, les effets de sens itératifs sont le résultat d'un ensemble des éléments du co(n)texte.

Cette approche nous permet de mieux observer la production de l'itération dans l'époque passée en français qui est l'objectif principal de ce travail. Dans les pages qui suivent, nous allons montrer comment le français peut exprimer l'itération passée : la participation de l'imparfait, du passé composé, du passé simple voire du plus-que-parfait dans la production de l'itération, quelles différences existent dans la production de l'itération parmi ces temps verbaux et quels sont les équivalents sémantiques serbes exprimant la répétition d'une action dans le passé et vice versa. Il faut mentionner ici qu'en ce qui concerne la langue serbe, les formes verbales liées au phénomène de l'itération dans le passé sont le parfait des verbes imperfectifs et le potentiel (PIPER, ANTONIĆ et al. 2005, IVIĆ 2008). Notre analyse du corpus d'une

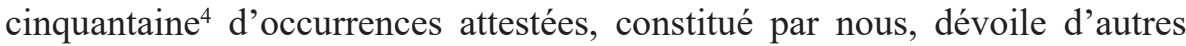
possibilités dans le cadre de la traduction des formes verbales exprimant l'itération passée dans les deux langues.

\section{L'expression d'itération dans l'époque passée en français}

Selon Bres (2007), pour pouvoir analyser le rôle d'un temps verbal dans la production de l'itération, il faut d'abord définir sa valeur en langue, i.e. déterminer ses instructions temporelles (situant un procès dans une des trois époques) et aspectuelles (concernant les catégories de la tension (i) et de l'incidence (ii) représentant le temps interne au procès) :

a) les instructions aspectuelles [+ tension] / [+ extension] permettent de marquer la différence entre formes simples et formes composées, i.e. les formes simples représentent le temps interne au procès en tension, entre les bornes initiale et terminale, et les formes composées représentent le temps interne au procès en extension, à partir de la borne terminale atteinte ;

b) les instructions aspectuelles [+ incidence] / [-incidence] « concernent principalement le mode d'inscription du temps interne impliqué par le verbe sur l'imaginaire ligne du temps que construit le discours » (BRES 2009 :

\footnotetext{
plus-que-parfait.

${ }^{4}$ Ledit nombre d'attestations s'explique par la rareté du passé simple et du plus-que-parfait itératifs. Pour la constitution de notre corpus, nous avons eu accès à la base de données Frantext et à la base de données (le corpus parallèle français-serbe) sur le site de la Faculté des Mathématiques (www.korpus.matf.bg.ac.rs).
} 
17) ; la première concerne les formes représentant le procès de façon sécante (cursive ou dans son cours), c'est-à-dire sans prendre en compte les bornes, tandis que la deuxième implique les formes produisant une vision globale du procès.

Distinguer les temps verbaux du passé mentionnés supra dans le cadre du paradigme aspectuo-temporel nous permettra également de faire la différence entre deux types d'itération, à savoir l'itération cursive et l'itération globale, dont il sera question infra.

\subsection{L'imparfait et l'itération passée}

Comme déjà mentionné supra le temps verbal typique pour exprimer l'itération en français est l'imparfait :

Chaque matin Paul allait chez le boulanger.

Cependant, en appui sur le travail de Bres (2007) nous considérons que l'itération n'est pas un sens de base de ce temps verbal, mais le résultat de la congruence de plusieurs facteurs : dans l'exemple supra il s'agit du type de verbe au sens vendlerien et de la présence d'un adverbe temporel (chaque matin).

Ensuite, dans le paradigme aspectuo-temporel, l'imparfait représente un des temps verbaux de l'indicatif situant le procès dans l'époque passée et il possède les instructions aspectuelles suivantes : [+ tension], [- incidence]. Cela signifie que l'imparfait en tant qu'une forme simple représente le procès entre les bornes initiale et terminale et il donne à voir le procès dans son cours. Quel est alors le rôle de l'imparfait dans la production de l'itération? Selon tous les faits mentionnés supra nous pourrions conclure que l'imparfait participe dans la production de l'itération passée cursive.

\subsection{Le passé composé et l'itération passée}

Nous avons déjà mentionné la possibilité de désigner l'itération dans l'époque passée par le passé composé. Ainsi, dans l'exemple déjà mentionné supra:

Longtemps, je me suis couché de bonne heure.

le passé composé en combinaison avec le co(n)texte où l'adverbe longtemps signifie en fait souvent et non pendant un $x$ temps (du fait que la longueur du procès est sentie comme incompatible avec le passé composé) désigne l'itération.

Nous ajouterons la description de ce temps verbal de l'indicatif dans le cadre du paradigme aspectuo-temporel qui est la suivante : [+ tension], [+/incidence]. On peut remarquer ici que l'unique différence entre l'imparfait 
et le passé composé se trouve dans la représentation aspectuelle du passé composé qui est neutre. Cela signifie que le passé composé marque le procès globalement ou cursivement selon le co(n)texte, i.e. il peut marquer l'itération globale ou cursive en fonction du co(n)texte. Maintenant il est possible d'expliquer plus précisément l'interprétation itérative de l'exemple supra: le passé composé à l'aide du co(n)texte - la présence de l'adverbe longtemps, le type du verbe au sens vendlerien indiquant que le procès entier est vu dans son cours - construit l'itération cursive, tandis que dans le cas suivant le passé composé participe dans la production de l'itération globale (moyennant l'adverbe souvent, le type du verbe au sens vendlerien se souvenir de) :

(3) Je me suis souvent souvenu de ma mère.

\subsection{Le passé simple et l'itération passée}

Il est bien notoire que le passé simple est défini en opposition à l'imparfait. Dans toute la littérature est fait mention que c'est un temps verbal de l'indicatif présentant les procès brefs, achevés et successifs dans le passé alors que l'imparfait représente les procès qui durent, qui sont inachevés et qui peuvent se répéter dans le passé. Soulignons que nulle part n'est mentionné la possibilité du passé simple marquant l'itération passée.

Cependant, c'est Bres (2007 : 43) qui affirme que le passé simple peut participer dans la production de l'itération. Comme l'imparfait, le passé simple est un temps verbal de l'indicatif situant le procès dans le passé, possédant la forme simple ce qui signifie qu'il représente le procès entre les bornes initiale et terminale. D'après ses instructions aspectuelles [+ tension], [+ incidence], le passé simple donne à voir le procès en accomplissement (de sa borne initiale à sa borne finale). Ainsi, par rapport à l'imparfait, le passé simple peut participer dans la production de l'itération globale.

Cette possibilité du passé simple n'est point étonnant car ce temps verbal, utilisé avec des verbes atéliques donne l'interprétation globale :

(4) Il marcha de 10 à 11 heures.

Cette lecture est en fait le résultat du conflit entre les aspects grammatical et lexical où l'aspect grammatical prévaut. Ainsi dans l'exemple supra le verbe imperfectif marcher obtient une lecture perfective : on appelle ce processus la coercion aspectuelle ${ }^{5}$ (STANOJEVIĆ, AŠIĆ $2006: 34$ ).

Regardons l'exemple suivant :

(5) Alors, pendant une semaine, Rosalie fit chaque jour un voyage à Fécamp

${ }^{5}$ Le mécanisme de la coercion aspectuelle est déclenché quand il est en vigueur un conflit entre l'aspect lexical et l'aspect grammatical. Dans ce cas, le passé simple, combiné à un verbe imperfectif, exige la transformation aspectuelle du verbe imperfectif en une éventualité perfective. 
pour se faire expliquer les choses par un notaire qu'elle connaissait. (G.de

Maupassant, Une Vie)

On peut remarquer que le passé simple est intégré à la saisie itérative du procès faire marquée par l'instruction des circonstants chaque jour et pendant une semaine. Soulignons également que la présence de l'adverbe pendant une semaine demande la lecture globale de l'exemple (5) et on a donc affaire à l'itération globale.

\subsection{Le plus-que-parfait et l'itération passée}

Si la présentation du passé simple dans le cadre de l'itération était un fait intéressant, la capacité du plus-que-parfait de marquer l'itération dans le passé représenterait la donnée encore plus remarquable.

Il s'agit d'un temps verbal de l'indicatif situant le procès dans l'époque passé (son instruction temporelle), mais, à la différence de l'imparfait et du passé simple, ce temps est morphologiquement la forme composée signifiant qu'il relève de l'aspect extensif. En effet, selon son instruction aspectuelle [+ extension], le plus-que-parfait représente le procès à partir de la borne terminale atteinte, apportant alors le sens d'accompli.

Comment est-il possible que ce temps verbal puisse marquer l'itération ? Bres (2007 : 48) l'affirme : « C'est donc non pas le $\mathrm{PqP}^{6}$ qui est allergique à la fréquence itérative ; mais le $\mathrm{PqP}$ dans un cotexte spécifique. » Il est possible alors de construire l'itération passée avec le plus-que-parfait dans un co(n)texte approprié ; quel type ? Son instruction aspectuelle concernant la catégorie de l'incidence est la suivante : [- incidence], comme celle de l'imparfait, indiquant que le plus-que-parfait représente le procès dans son cours, ce qui implique qu'il peut produire l'itération cursive.

Dans le cas de (6) on peut remarquer que le plus-que-parfait déclenche la lecture itérative cursive :

(6) Il avait également ressenti un trouble vague chaque fois qu'elle s'était penchée vers lui ou qu'elle l'avait regardé fixement de ses yeux gris. (N. Roberts, La saga des Stanislaski)

Les procès pencher et regarder marqués par les plus-que-parfaits $s$ 'était penchée et avait regardé sont représentés à partir de leur borne terminale atteinte ; la conjonction chaque fois que met en relation de succession immédiate l'atteinte de cette borne avec le procès qui suit (ressentir). Ainsi, le plus-que-parfait s'accorde ici parfaitement avec la saisie cursive de l'itération. Soulignons aussi que la conjonction temporelle chaque fois que dont l'instruction primaire est la progression temporelle immédiate entre les actions de la principale et de la subordonnée, signifie aussi la répétition

${ }^{6}$ Bres utilise cette abréviation pour marquer le plus-que-parfait. 
et le caractère habituel d'une action préparant ainsi le co(n)texte pour l'interprétation itérative.

\section{Question de la traduction des formes verbales désignant l'itération passée en français}

Dans le cadre du domaine de la traductologie, auquel nous portons de l'intérêt aussi dans ce travail, il faut mentionner que tous les linguistes franco-serbes soulignent que l'équivalent serbe par excellence de l'imparfait exprimant l'itération dans le passé est le parfait des verbes imperfectifs :

(7) ...il se promenait un peu chaque jour dans le jardin de la préfecture. (Mémoires du cardinal Barthélémi Pacca)

(7a) ....̌etao se malo svakog dana u bašti prefekture. (www.korpus.mat.bg.ac.rs)

L’exemple (7) décrit un état de chose qui était valable dans le passé. Le procès se promener en $(7,7 \mathrm{a})$ actualisé à 1 'imparfait et au parfait, est représenté itérativement, comme l'explicite le circonstant chaque jour. C'est le cotexte (le type du verbe au sens vendlarien, le circonstant mentionné) qui indique que l'habitude, i.e. la pratique quotidienne du cardinal, est saisie dans son cours ce qui sélectionne l'imparfait en français et le parfait imperfectif en serbe.

Comme nous l'avons mentionné en introduction, dans la langue serbe on peut utiliser deux formes verbales marquant l'itération d'un procès dans le passé, à savoir le parfait imperfectif et le potentiel. Ainsi l'autre possibilité de traduire l'imparfait français produisant l'itération passée est d'employer le potentiel en serbe comme on peut le remarquer infra:

(7b) ...(pro)šetao bi se malo svakog dana u bašti prefekture.

Y-a-t-il une différence entre lesdites formes verbales dans la production de l'itération passée ? Bien évidemment, mais ce sujet sera développé dans nos futures recherches. Ici, nous allons résumer brièvement l'explication. D'abord, il s'agit d'une différence au niveau stylistique entre ces formes verbales. Selon Ivić (2008 : 60), leur emploi est fondé sur 1'opposition : « l'information strictement factuelle de la répétition de l'action - la représentation émotionnelle de l'action » où le parfait figure dans le premier cas et le potentiel dans le deuxième. Ensuite, l'opposition entre le parfait imperfectif et le potentiel relève de la représentation des événements itérés : avec le premier on décrit un état des choses dans le passé (exemple 7a) alors qu'avec le deuxième on insiste sur chaque occurrence singulière de l'événement (exemple 7b).

Il faut ajouter aussi que le serbe, par rapport au français, ne marque pas linguistiquement la différence entre l'itération globale et cursive. Le parfait 
construit l'itération cursive du fait qu'il donne à voir le procès dans son cours se trouvant uniquement en combinaison avec les verbes imperfectifs (7a), tandis que le potentiel peut produire les deux types de l'itération (cursive et globale) étant donné qu'il entre en combinaison avec les deux types aspectuels d'un verbe, à savoir perfectifs (prošetati se 's'être promené') et imperfectifs (šetati se 'se promener', 7b).

S'agissant de la traduction du passé composé, on peut inférer la même conclusion réservée à l'imparfait, à savoir le passé composé est traduit en serbe soit par le potentiel soit par le parfait imperfectif. Du fait que selon son instruction aspectuelle et selon le co(n)texte, le passé composé peut participer dans la production de l'itération soit globale soit cursive, il se traduit en serbe moyennant le potentiel perfectif s'il faut désigner l'itération globale :

(8) - ...Je ne t'ai jamais oublié...je songeais à toi sur la route de Sens...tu sais... - Ah...je crois bien que je sais !...En Afrique, mille fois, je me suis souvenu de cette nuit et de cette auberge à l'enseigne de la Belle-Étoile... (J. J. Grandville, Les Étoiles)

(8a) -...Nikada te nisam zaboravio...mislio sam na tebe tokom puta ka Sensi... znaš... - Pa znam! ...Hiljadu puta bih se u Africi setio te noći i gostionice Lepa Zvezda... (www.korpus.mat.bg.ac.rs)

ou le parfait / le potentiel imperfectifs pour marquer l'itération cursive (sans (9a) ou avec les éléments stylistiques - (9b) :

(9) Je me suis demandé parfois si un trop grand amour ne paralysait pas, en nous, les forces d'action... (Revue des deux mondes)

(9a) Pitao sam se ponekad da li tako velika velika ljubav može da parališe snagu u nama... (www.korpus.mat.bg.ac.rs)

(9b) Pitao bih se ponekad da li tako velika velika ljubav može da parališe snagu u nama...

La question qui s'impose est comment traduire le passé simple désignant l'itération passée. Disons tout d'abord que l'aoriste serbe ne peut pas avoir cette fonction :

(10) L'avion revint presque chaque jour pendant une semaine.

(10a) *Avion se vrati svakog dana tokom jedne sedmice.

Pourquoi son emploi n'est-il pas de mise? Par rapport au passé simple, l'aoriste serbe est très rarement employé dans la narration. Mentionnons-en que l'aspect grammatical, marqué par l'opposition aoriste/imparfait, était pertinent en serbe, mais l'aspect morphologique a pris la prépondérance. Ainsi, l'imparfait est devenu superflu dans le système verbal serbe et l'aoriste est tombé partiellement en désuétude dans la langue serbe contemporaine. 
Cependant, il s'utilise très souvent en langue quotidienne pour désigner un procès situé juste avant le moment de la parole :

$\begin{array}{lll}\text { Sad } & \text { ode } & \text { kući. } \\ \text { maintenant } & \text { partit } & \text { à maison }\end{array}$

Il vient de partir chez lui.

À la différence de l'aoriste serbe, le passé simple français marque un procès passé qui est détaché du moment de la parole et ce fait aussi explique l'impossibilité d'employer l'aoriste comme l'équivalent du passé simple.

La première traduction du passé simple qui nous vient à l'esprit est celle du parfait imperfectif :

(11) Vers la mi-janvier, après des chutes de neige qui rendirent les chemins tout à fait impraticables, le temps s'éclaircit et un avion de reconnaissance allemand, à l'heure du déjeuner, remonta la vallée de la Meuse. [...] Le spectacle ne parut à Grange nullement guerrier [...] L'avion revint presque chaque jour pendant une semaine. Grange pensa que la neige rendait les terrassements en cours sur la position de la Meuse plus visibles. (J. Gracq, Un Balcon en forêt)

(11a) Oko polovine januara nakon snežnih padavina koje su zavejale puteve vreme se prolepšalo i jedan nemački avion u vreme ručka pojavi se iznad doline Mez. Taj prizor nije delovao Granžu nimalo ratnički. Avion se vraćao svakog dana tokom jedne sedmice. Granž pomisli kako sneg čini vidiljivljim zemljane radove na poziciji Meza.

Dans l'exemple (11) les procès actualisés au passé simple : s'éclaircit, remonta, parut, revint se suivent et on remarque par l'instruction des circonstants chaque jour et pendant une semaine que le dernier procès au passé simple construit l'itération globale. Cependant, avec le parfait dans l'équivalent serbe (11a) on perd la progression temporelle ${ }^{7}$. En plus, on ne peut pas désigner l'itération globale à l'aide du parfait imperfectif. Ainsi, la traduction de l'exemple français moyennant le parfait imperfectif ne représente pas la meilleure solution.

Il nous reste à considérer la traduction à l'aide du potentiel. Nous avons remarqué, dans notre corpus, que la meilleure solution de traduire le passé simple introduisant l'itération passée est d'employer le potentiel des verbes perfectifs :

(11b) Avion bi se vratio svakog dana tokom jedne sedmice.

\footnotetext{
${ }^{7}$ Rappelons que, de par son instruction discursive [+ neutre], le parfait imperfectif serbe peut entrer dans toutes relations discursives, à savoir simultanéité, regression et progression. L'inférence de tel ou tel type de relation dépend de nos connaissances du monde ou du co(n)texte. À la différence du parfait imperfectif serbe, le passé simple français introduit la progression temporelle de deux événements ce qui dicte son instruction discursive de base $[+$ progression].
} 
étant donné qu'avec avec le potentiel perfectif la progression temporelle entre les procès est assurée. Ce fait est plus évident dans le cas suivant :

(12) Parfois, ils sentirent tout à coup un frisson et comme le vent d'une idée ; au moment de la saisir, elle disparut. (G. Flaubert, Bouvard et Pécuchet)

(12a) Ponekad bi osetili drhtaj i kao dah neke ideje; taman bi je zgrabili, a ona bi nestala. (www.korpus.matf.bg.ac.rs)

Ici le passé simple est employé pour désigner l'itération mais aussi pour souligner la présence de la progression temporelle : sentirent $<$ disparut. En serbe cela s'atteint parfaitement moyennant le potentiel perfectif. Ajoutons que la présence de la conjonction temporelle perfective taman ('dès que') bloque l'emploi du parfait serbe imperfectif.

Dans les exemples suivants il ne s'agit pas d'itération globale : le passé simple y est employé pour vivifier les événements devant les yeux du lecteur.

(13) Le nouveau scheik gouverna comme avait fait son prédécesseur, et fit les mêmes disparitions au dernier vendredi de chaque lune. (Jan Potocki, Oeuvres) (13a) Novi šeik vladao je kao i njegov prethodnik i isto tako bi iščeznuo poslednjeg petka svakog meseca. (www.korpus.matf.bg.ac.rs)

Ici, il ne s'agit pas d'un état de choses mais d'un événement particulier qui se répétait tous les derniers vendredis. Le choix du passé simple s'explique par le fait que l'itération est bornée à gauche : la montée au pouvoir du nouveau scheik. En serbe, l'effet stylistique mentionné supra est exprimé en serbe par le potentiel, qui se perd si on utilise le parfait.

Dans l'exemple (14) il n'y a pas de borne mais le narrateur veut insister sur chaque occurrence particulière de l'événement :

(14) Mon père se confessa au Père Geronimo tous les derniers dimanches de chaque mois. (Jean Potocki, Voyage lointain)

(14a) Moj otac bi se ispovedio kod oca Heronima poslednje nedelje svakog meseca. (www.korpus.matf.bg.ac.rs)

Ici, on a l'impresson de participer à la confession ce qui relève de l'emploi du passé simple et du potentiel perfectif. On insiste sur chaque réalisation de l'événement en question.

Quant à la traduction du plus-que-parfait, nous avons eu beaucoup de difficultés de trouver des occurrences attestées ce qui s'explique par la rareté du plus-que-parfait participant dans la production de l'itération passée. En appui sur le corpus de Bres (2007) et sur l'aide du traducteur-interprète assermenté près le Tribunal de Grande Instance de Belgrade, Vladimir Pavlović, nous avons déterminé que l'équivalent serbe (le plus fréquent) du plus-que-parfait en question et figurant dans les subordonnées temporelles est le potentiel. 
Dans le cas de (15) le plus-que-parfait, se trouvant dans la subordonnée temporelle introduite par dès que indiquant l'itération, s'intègre à l'itération cursive à l'imparfait même s'il y apporte aussi le sens d'accompli selon son instruction [+ extension]. Cela explique pourquoi il est traduit par le potentiel parfait dans l'exemple (15a).

(15) Dès que Julia avait servi le thé, ils se dévêtaient dans une hâte anxieuse ; dans la grande pièce assombrie, toute pleine de la tristesse paysanne du soir, ils s'étreignaient sans parler. (J. Gracq, Un Balcon en forêt)

(15a) Čim bi Julija poslužila čaj, oni bi se užurbano i nervozno skidali; u velikoj, zamračenoj prostoriji, punoj večernje seljačke tuge, grlili bi se ne govoreći.

Le plus-que-parfait peut figurer en indépendantes produisant l'itération passée. Cependant, nous avons dû retravailler son occurrence issue du roman de J. Gracq. Dans ce cas, on peut remarquer qu'il peut être traduit en serbe par les deux formes verbales désignant l'itération passée, à savoir par le potentiel (16a) et par le parfait imperfectif (16b) :

(16) La semaine précédente, tous les jours en début d'après-midi, Grange et Olivon s'étaient assis un peu en retrait du bord de la route ; ils avaient regardé passer les blindés.

(16a) Prošle nedelje, svakog dana u rano popodne, Granž i Olivon bi zaseli malo dalje pokraj puta; posmatrali bi tenkove kako prolaze.

(16b) Prošle nedelje, svakog dana u rano popodne, Granž i Olivon su posedeli malo dalje pokraj puta; posmatrali su tenkove kako prolaze.

La différence dans la traduction a été déjà expliquée supra et elle concerne la production des effets stylistiques et la manière de la représentation des événements.

\section{Question de la traduction des formes verbales désignant l'itération passée en serbe}

La littérature traditionnelle nous apprend que le potentiel marquant l'itération dans le passé se traduit typiquement par l'imparfait français. Dodig traite également ce sujet dans son travail de 2018 :

(17) A ja bih ponekad ustao samo da prohodam po sobi, i opet legao. (M. Selimović, Tvrđava)

(17a) Je me levais parfois pour faire quelques pas dans la chambre, puis je me recouchais. (M. Selimović, La Forteresse) 
Cependant, c'est Dodig (2018 : 352-353) qui démontre le fait que l'emploi du passé simple comme équivalent du potentiel dénotant l'itération est également possible :

(18) Počevši od nedelje, svakog dana $\boldsymbol{b i}$ sebe terao malo dalje, svakog dana $\boldsymbol{b i}$ pokušao da vozi malo duže nego prethodnog dana. (corpus Dodig, 2018 : 352)

(18a) À partir du dimanche, chaque jour, il s'efforça d'aller un peu plus loin, chaque jour, il essaya de rouler plus longtemps que la veille.

Le choix du passé simple est motivé par la présence du circonstanciel $\grave{a}$ partir du dimanche qui borne l'itération à gauche.

Dans le cas suivant, l'équivalent français du potentiel est l'imparfait :

(19) I tako svakog dana isto : ja bih je ǐšcekivao, ona bi došla, moj otac bi se sakrio, a ja bih lagao na vratima. (S.Matavulj, Pripovetke)

(19a) Chaque jour la même chose se reproduisit : je l'attendais, elle arrivait, mon père se cachait et moi je mentais devant la porte. (www.korpus.mat.bg.ac.rs)

Cependant, nous sommes d'avis que l'on peut utiliser le passé simple du fait que la progression temporelle est en vigueur. Aussi, on participe à travers de la représentation du narrateur à chaque occurrence singulière des événements en question.

Dans le cas de (20), le choix du passé simple comme équivalent du potentiel s'explique par la présence du circonstant ponctuel trois fois par jour :

(20) Tog leta, njegova žena, koja je bila ljubomorna do besnila, tukla bi me nemilosrdno tri puta na dan.

(20a) Cet été, sa femme, qui était jalouse à la rage, me battit impitoyablement trois fois par jour. (www.korpus.mat.bg.ac.rs)

Dans l'exemple suivant nous avons affaire à l'itération globale grâce à la présence du circonstant temporel tokom pola veka ('pendant cinquante ans'), qui est marquée, comme attendu, moyennant le potentiel en serbe et le passé simple en français :

(21) Kada je prošle godine Koka-kola poklonila Kongresnoj biblioteci reklamne spotove kojima je, tokom pedeset godina, lansirano njeno čuveno piće svuda u svetu, pokazalo se da ih je bilo više od dvadeset hiljada. Tokom pola veka, svakog dana bi bio napravljen bar jedan spot ove kompanije.

(21a) Quand, l'année dernière, Coca - Cola fit don à la bibliothèque du Congrès de l'équivalent d'un demi - siècle de ses spots publicitaires diffusés dans le monde, il y en avait plus de vingt mille. En moyenne au moins un spot Coca Cola fut conçu chaque jour depuis cinquante ans. (www.korpus.mat.bg.ac.rs) 
S'agissant de la traduction du parfait imperfectif désignant l'itération passée, il est normalement traduit par l'imparfait français :

(22) Satima sam sjedio pred Begovom džamijom, na kamenu, i gledao ljude kako prolaze, ili nebo, ili ništa. Slušao sam vrapce i njihovo smiješno čavrljanje, kao u dobrodušnoj svađi, ili u vedrom pričanju o svemu i svačemu...Bili su pitomi i bezopasni, kao djeca. I djecu sam volio, njihove zvonke glasove, brzi topot bosih nogu...Samo, kad bi se potukli, zatvarao sam oči i uši, uznemiren. (M. Selimović, Tvrđava)

(22a) Je restais des heures entières assis sur une pierre devant la mosquée du Bey, à regarder les passants ou le ciel ou rien du tout. J'écoutais le pépiement cocasse des moineaux, leurs querelles amicales ou leur joyeux babillage... Familiers et inoffensifs comme des enfants. J'aimais aussi les enfants, leurs cris, la course rapide de leurs pieds nus...Ce n'est que lorsqu'ils se battaient que, troublé, je fermais les yeux et me bouchais les oreilles. (M. Selimović, La Forteresse)

Cela s'explique par le fait que les deux temps verbaux construisent l'itération cursive. Nous avons déjà expliqué comment l'imparfait peut participer dans la production de l'itération en question. Le sujet de l'interprétation itérative cursive dans le passé inférée par le parfait imperfectif reste à être abordé plus en détails dans nos futures recherches. Nous allons résumer ici seulement que l'itération cursive s'infère moyennant la nature atélique des verbes imperfectifs au parfait, les circonstants temporels fonctionnant comme marqueurs de l'itération ou simplement à l'aide du co(n) texte approprié, ce que l'on peut remarquer en (22).

\section{Conclusion}

Notre travail a permis de démontrer comment l'itération dans l'époque passée peut être exprimée en français. Plus précisément, nous avons traité les temps verbaux du passé : l'imparfait, le passé composé, le passé simple et le plus-que-parfait désignant l'itération. Nous avons attiré l'attention surtout sur certains temps verbaux du passé, comme le passé simple et le plus-queparfait, dont le sémantisme de base, à première vue, empêche la possibilité de produire l'itération. Ce travail a permis aussi de distinguer l'itération globale, à laquelle participe le passé simple (et le passé composé) de l'itération cursive que construisent l'imparfait et le plus-que-parfait (et le passé composé).

Finalement, nous avons prêté attention au domaine de la traductologie concernant l'itération passée en français et en serbe ce qui nous a dévoilé de nouveaux faits relevant de la traduction en question. Ainsi, nous avons vu que les équivalents serbes des temps verbaux du passé mentionnés supra peuvent être soit le potentiel soit le parfait imperfectif dont l'emploi dépend du besoin de styliser une action ou de la manière de la représentation des événements; 
ensuite, que le potentiel serbe désignant l'itération peut être traduit non seulement par l'imparfait français mais aussi par le passé simple français.

Reste à développer la partie concernant la traductologie ce que nous allons faire dans nos futures recherches.

\section{Bibliographie}

APOTHÉLOZ 2005 : APOTHÉLOZ, Denis. " RE- et les différentes manifestations de l'itérativité ». Pratiques : linguistique, littérature, didactique $\mathrm{n}^{\circ} 125-126$ (2005) : 48-71.

AŠIĆ 2007 : AŠIĆ, Tijana. „S one strane potencijala - novi pristupi objašnjenju upotrebe potencijala za označavanje ponavljanja u prošlosti ". Južnoslovenski filolog 36 (2007) : 137-150.

BRES 2007 : BRES, Jacques. « Fréquence narrative et temps verbal : une approche linguistique à partir de Un Balcon en forêt », L'Information grammaticale 115 (2007) : 42-49.

DODIG 2018 : DODIG, Milana. Le conditionnel français et ses équivalents sémantiques en serbe - étude comparative entre le conditionnel français et le potentiel serbe, Thèse de doctorat, Université Montpellier 3, Université de Kragujevac, 2018.

GOSSELIN, MATHET et al. 2013 : GOSSELIN, Laurent et Yann MATHET, Patrice ENJALBERT, Gérard BÉCHER. Aspects de l'iteration : l'expression de la répétition en français : analyse linguistique et formalisation. Bern : Peter Lang, 2013.

IVIĆ 2008 : IVIĆ, Milka. Lingvistički ogledi. Beograd : Biblioteka XX vek, 2008.

PIPER, ANTONIĆ et al. 2005 : PIPER, Predrag i Ivana ANTONIĆ, Vladislava RUŽIĆ, Sreto TANASIĆ, Ljudmila POPOVIĆ, Branko TOŠOVIĆ. Sintaksa savremenoga srpskog jezika. Prosta rečenica. Beograd: Institut za srpski jezik SANU - Beogradska knjiga - Matica srpska, 2005. [orig.] ПИПЕР, Предраг и Ивана АНТОНИЋ, Владислава РУЖИЋ, Срето ТАНАСИЋ, Људмила ПОПОВИЋ, Бранко ТОШОВИЋ. Синтакса савременога српског језика. Проста реченища. Београд: Институт за српски језик САНУ - Београдска књига - Матица српска, 2005.

RIEGEL, PELLAT et al. 1994 : RIEGEL, Martin et Jean-Christophe PELLAT, René Rioul. Grammaire méthodique du français. Paris : Presses universitaires de France, 1994.

STANOJEVIĆ, AŠIĆ 2006: STANOJEVIĆ, Veran et Tijana, AŠIĆ. Semantika i pragmatika glagolskih vremena u francuskom jeziku. Kragujevac : Filološko-umetnički fakultet, 2006.

WILMET 2003: WILMET, Marc. Grammaire critique du français. Paris : Hachette supérieur, 2003. 
Милана Л. Додиг

\section{ИЗРАЖАВАЬЕ ИТЕРАЦИЈЕ У ПРОШЛОСТИ У ФРАНЦУСКОМ ЈЕЗИКУ СА ОСВРТОМ НА ЮЕН ПРЕВОД}

У овом раду посветили смо пажњу питању статуса итерације уопште, с посебним освртом на изворе, тј. маркере итерације. Наш главни циљ био је да представимо и анализирамо начине изражавања понављања једног догађаја у прошлости у француском језику. Такође, бавили смо се и питањем превођења итерације у прошлости у француском и у српском језику на основу корпуса који смо конституисали. Овај приступ нам је омогућио да откријемо нове језичке чињенице, које се тичу саме итерације и итерације у прошлости, док је анализа нашег корпуса указала и на друге могућности изражавања итерације у прошлости, о чему до сада није било речи у литератури.

Кључне речи: итерација, прошлост, аспект, време, превод 